\title{
Estratégias para os Elementos Culturais na Tradução
}

Resumo: Tradução é um processo de reescrita, manipulação, comunicação e mediação intercultural. Os elementos culturais trazem grandes desafios para um tradutor, e as estratégias para lidar com estes elementos são dependentes dos contextos envolvidos. São escolhidos neste artigo alguns exemplos de quatro perspectivas na tradução de inglês para chinês do livro intitulado The Poetry of Edwin Morgan, escrito por James McGonigal, com o objetivo de demonstrar as estratégias adotadas no processo da tradução como tradutora/mediadora. $\mathrm{O}$ artigo ilustra as mudanças funcionais que ocorrem quando um guia para uma figura literária escocesa de importância nacional num contexto específico é traduzido para um público menos familiarizado com a sua obra.

Palavras-chave: estudos de tradução; elementos culturais; estratégias de tradução; escrita; comunicação

\begin{abstract}
Translation is a process of rewriting, manipulation, communication, and intercultural mediation. Cultural elements constitute great challenges for a translator and strategies for dealing with these elements are dependent on the contexts involved. Some examples are chosen from four perspectives in the Chinese translation of the English book entitled The Poetry of Edwin Morgan written by James McGonigal to demonstrate the strategies adopted in the translation process as a translator / mediator. The article illustrates the functional changes that occurred when a guide to a literary figure of national importance in one particular context was translated for an audience that is less familiar with his oeuvre.
\end{abstract}

Key Words: Translation Studies; cultural elements; translation strategies; rewriting; communication

1 Li Li é tradutora e Professora Titular na Escola Superior de Línguas e Tradução do Instituto Politécnico de Macau (IPM). Doutorou-se em Estudos de Tradução (Inglês-Chinês) pela Universidade Chinesa de Hong Kong (2006) e obteve o grau de mestrado em Estudos de Tradução (Inglês-Chinês) pela Universidade de Estudos Estrangeiros de Guangdong na China (2003). Atualmente é mestranda em Tradução e Interpretação Chinês-Português no IPM. Contato: lili@ipm.edu.mo 


\section{Introdução}

Os conhecimentos sobre a definição de tradução, o papel de tradutor e os conceitos de cultura desempenham um papel significativo para um tradutor na escolha das estratégias adequadas num processo de tradução. A definição de tradução tem mudado ao longo do tempo. Hoje em dia, tradução não é apenas um processo de transferência linguística, mas um processo de reescrita, manipulação, comunicação e mediação intercultural com skopos (LEFEVERE, 2006/2016; HERMANS, 1985; NORD, 2001; KELLY, 2005; MUNDAY, 2016). Correspondentemente, o papel de tradutor mudou de transmissor linguístico para mediador intercultural (KATAN,1999). Muitas categorizações de cultura foram feitas na área de tradução (NEWMARK, 1988; BAKER, 1992; KATAN ,1999); por exemplo, Newmark (1988, p. 21) listou cinco categorias:

- ecologia (flora, fauna, ventos, clima, etc.);

- cultura material (comida, roupa, casas, cidades, transporte);

- cultura social (trabalho e lazer);

- organizações, costumes, atividades, procedimentos ou conceitos (que incluem arte, religião, subcategorias políticas e administrativas);

- gestos e hábitos.

Estas categorias podem ajudar um tradutor a identificar as barreiras culturais que prejudicam a compreensão dos leitores-alvo se os elementos culturais forem traduzidas literalmente. Essas barreiras culturais são evidentes quando um guia introdutório para a poesia de uma figura de importância nacional num país específico, neste caso o poeta escocês Edwin Morgan, é traduzido para um público menos familiarizado com o seu trabalho, designadamente o público chinês.

Edwin Morgan (1920-2010), poeta e tradutor escocês, é amplamente reconhecido como um dos principais poetas escoceses do século XX. Em 1999, Morgan foi nomeado o primeiro 'Poeta Laureado' de Glasgow e em 2004 'The Scots Makar', o primeiro poeta nacional escocês. Os temas e estilos da sua poesia são diversificados.

The Poetry of Edwin Morgan é um livro escrito por James McGonigal (1947-) e publicado pela Associação para Estudos Literários Escoceses em 2013 como livro guia de estudo orientado principalmente para os estudantes secundários, os seus professores e os estudantes universitários na Escócia. Esse livro, em conjunto 
com outros quatro livros salientando autores como por exemplo Robert Louis Stevenson (1850-1894), Muriel Spark (1918-2006), Robert Burns (1759-1796), e baladas escocesas (The Scottish Ballads), foi escolhido para ser traduzido para o chinês e será publicado em 2020 pela Imprensa de Publicação da Universidade de Nanjing na China. A autora deste artigo foi convidada a traduzir o livro The Poetry of Edwin Morgan do inglês para chinês com a principal finalidade de promover os conhecimentos dos chineses sobre literatura e cultura escocesas na China. Em vista da falta de conhecimento do povo chinês em geral sobre a literatura e cultura escocesas, bem como a diversidade e complexidade do próprio poeta Edwin Morgan, o processo de tradução foi abundante em desafios para a tradutora. Na parte seguinte deste artigo, as estratégias tradutórias são ilustradas para demonstrar meus esforços com vistas a realizar uma tradução mais compreensível para os leitores chineses através da manipulação, comunicação e reescrita.

\section{Estratégias no processo da tradução}

Muitos investigadores propuseram estratégias para lidar com os elementos culturais (CHESTERMAN, 1997; BAKER, 1992; NORD, 2001). Por exemplo, Nord (2006, p. 53) listou duas opções relacionadas com a função referencial na tradução quando a intenção referencial do remetente do texto de origem não pode ser transmitida, a saber, a oferta de informações adicionais sobre a situação do texto de partida num metatexto (como nota de rodapé, glossário, prefácio), e a expansão no texto ou a transformação das informações implícitas do texto fonte numa informação textual explícita. As estratégias discutidas neste artigo são diretamente ou indiretamente relacionadas com essas duas opções.

\subsection{Caraterísticas naturais}

Nesta parte, dois aspectos da natureza são selecionados, nomeadamente, os locais, e as plantas e os animais, para demonstrar as estratégias de tradução correspondentes.

\subsubsection{Os locais}

Os locais e as cidades são uma parte importante de uma cultura, e os nomes dos locais, quando transferidos para outra cultura, podem causar dificuldades para os leitores desconhecedores do local. No livro The Poetry of Edwin Morgan, James 
McGonigal mencionou no primeiro capítulo dois sítios que tinham importância na vida de Edwin Morgan:

Crescer em Rutherglen e Glasgow colocou-o [Edwin Morgan] em contacto com aspectos da vida aos quais ele voltaria constantemente na sua escrita. ${ }^{2}$ (MCGONIGAL, 2013, p. 6)

Nesta frase existem duas localidades na Escócia: uma vila Rutherglen e uma cidade chamada Glasgow. Para os leitores escoceses, os nomes são conhecidos; contudo, se para os chineses, Glasgow, como a capital da Escócia, é conhecido na China, o nome de Rutherglen é algo de exótico e, sobretudo, pouco conhecido. Rutherglen é uma vila no sul da Escócia; além disso, também é uma vila no estado de Vitória na Austrália. Para os leitores chineses terem uma ideia geral sobre a localização de Rutherglen e Glasgow, a tradutora adicionou 'a Escócia' antes destes locais no corpo do texto, ou seja, uma expansão no texto, segundo Nord (2006).

Às vezes, os nomes das localidades têm significância associada com eles. Por exemplo, Sharpeville é uma cidade situada na província do Transvaal na África do Sul; sendo também o nome do poema que Edwin Morgan escreveu sobre o massacre racista ocorrido em 21 de março de 1961, no qual 69 pessoas foram mortas pela polícia local. Neste caso, o conhecimento sobre o massacre é essencial para perceber o significado do poema e, por isso, quando a cidade de Sharpeville foi mencionada, a tradutora adicionou a informação sobre o massacre como nota de rodapé na tradução, o que é a primeira das estratégias mencionadas por Nord (2006) para lidar com os elementos culturais na tradução.

Ainda que os dois exemplos pertençam à mesma categoria de lugares, as estratégias de tradução variam: uma adição informativa complementar no corpo do texto é suficiente no primeiro caso; porém, no segundo exemplo, uma nota de rodapé é necessária para uma compreensão mais profunda do conteúdo do poema envolvido.

2 O texto de partida: Growing up in Rutherglen and Glasgow brought him [Edwin Morgan] into contact with aspects of life that he would constantly return to in his writing. As traduções dos textos de partida citados são feitas pela autora deste artigo. 


\subsubsection{As plantas e os animais}

As plantas e os animais provenientes de um local específico podem formar fenômenos exclusivos e ter impactos nos sentimentos e provocar memórias especiais na população daquele local. Uma planta e um animal mencionados no livro serão usados aqui para demonstrar as estratégias relativas às respetivas traduções.

\section{As plantas: 'mistletoe' ('azevinho')}

São mencionadas nos poemas de Edwin Morgan muitas plantas, das quais escolhi um tipo: 'mistletoe' ('azevinho'). O raminho de azevinho aparece no poema intitulado 'Trio', descrevendo três pessoas (por isso 'trio' no título - duas mulheres e um homem) que passaram nas ruas a noite de Natal, cada pessoa trazendo alguma coisa simples, mas feliz. No caso do homem, uma guitarra nova decorada com um enfeite de prata e um raminho de azevinho. Hoje em dia, o Natal também se celebra na China, particularmente nas lojas para promover vendas, e o azevinho pode ser visto, mas o próprio nome e a sua associação e tradição ligadas com esta planta são desconhecidos para muitos chineses. O azevinho é uma planta parasítica e, ao longo do tempo, angariou muitos significados simbólicos, como por exemplo, fertilidade masculina, paz e felicidade, proteção contra bruxas e demônios, Jesus Cristo, vitalidade. ${ }^{3}$ O conhecimento sobre essa planta e os significados a ela associados podem ajudar os leitores a entender e interpretar o poema de uma forma mais profunda e multidimensional. No caso da autora que vos escreve, como leitora e tradutora, no início, antes de pesquisar as informações sobre esta planta, só a considerou como uma planta comum ou, quando muito, uma decoração comum para o Natal. Depois de ter lido as associações ligadas com a planta, consegui criar diferentes abordagens no processo de interpretação e compreensão deste poema. Acreditando que a própria experiência como leitora, mas simultaneamente como conhecedora da cultura envolvida, ajuda e enriquece a interpretação do poema, adicionei uma nota de rodapé como 'a oferta de informações adicionais sobre a situação do texto de partida', explicando brevemente os significados associados ao azevinho.

3 https://www.theholidayspot.com/christmas/history/mistletoe.htm. 


\section{A fauna: 'starlings' ('estorninhos') e snake ('serpente')}

Além das plantas, a fauna também é importante numa cultura. Dois tipos de pássaros e répteis, nomeadamente, os estorninhos e a serpente, são usados como exemplos. No livro, James McGonigal fala sobre um poema chamado 'The Starlings in George Square' ('Os Estorninhos na Praça George’):

'Os Estorninhos na Praça George' (a principal praça cívica de Glasgow) também abre com uma cena nocturna em que os estorninhos se precipitam em voo prestes a empoleirar-se: 'como uma chuva de setas eles atravessam / o clarão de uma janela ocidental, / e prendem os fios com jacto ...[....... $]^{44}$ (MCGONIGAL, 2013, p. 27)

Esta parte citada inclui dois elementos culturais: a ave 'starling' (estorninho) e 'George Square' (Praça George). O poeta criou este poema para descrever uma memória inesquecível como o rapaz no poema - 'ele nunca esquecerá aquela noite' (MORGAN, 1990, p. 165) e pensar a relação entre as aves e os seres humanos. Como leitora e tradutora sem muito conhecimento sobre aves, estorninhos neste caso, pesquisei as informações sobre este tipo de aves e fiquei a saber que os estorninhos no inverno antes de migrar para a Rússia, fazem estadia na Escócia, criando uma imagem espetacular. Muitas fotos e notícias sobre os estorninhos na Escócia são publicados na internet. Para os leitores chineses, com a deterioração do meio ambiente e as alterações climáticas, não é frequente ver a imagem de uma grande concentração de aves num mesmo espaço, por isso, não é fácil entender porquê da memória de Edwin Morgan, associada ao nome e à imagem de olhos bem abertos do rapaz no poema, tão impressionante e inesquecível que foi para ele vivenciar aquele momento. Levando isto em consideração, acrescentei uma nota de rodapé para explicar o fenômeno dos estorninhos na Escócia para os leitores chineses atingirem uma compreensão melhor do poema.

Mais um exemplo de fauna, 'snake' ('serpente'), será selecionado para explicar a estratégia de tradução. Baseado na caraterística do uso de consonantes 's' e ' $z$ ' da língua húngara, Edwin Morgan criou um poema concreto intitulado 'Siesta of a Hungarian Snake' ('Siesta de uma Serpente Húngara'), descrevendo a sesta de uma serpente após uma refeição satisfatória de uma forma vívida através do arranjo de letras grandes e pequenas:

4 O texto de partida: 'The Starlings in George Square' (Glasgow's main civic square) also opens on an evening scene as the starlings swoop down to roost: 'like a shower of arrows they cross/ the flash of a western window, / they bead the wires with jet [......]' 
Siesta of a Hungarian Snake

s sz sz SZ sz SZ sz ZS zs ZS zs zs z (MCGONIGAL, 2013, p. 17)

Queria citar aqui a tradução deste poema, do inglês para português, por Virna Teixeira (1971-), escritora, poeta e tradutora brasileira que mora em Londres. Como a autora deste artigo fez na sua tradução chinesa, só o título do poema foi traduzido, ou seja, a linha poética fica exatamente igual ao texto de partida:

Siesta de uma cobra húngara

s sz sz SZ sz SZ sz ZS zs ZS zs zs z (TEIXEIRA, 2006, p. 93)

A palavra 'cobra' no título na versão de Virna Teixeira é muito interessante. No poema inglês, Edwin Morgan usou a palavra 'snake', o que não especifica que tipo de serpente ele está a descrever, e James McGonigal entende que a serpente é víbora (rattlesnake). Mesmo que seja "muito comum as pessoas usarem no Brasil como sinônimos 'cobra', 'serpente', 'cascavel'... A palavra cobra muitas das vezes é utilizada como sinônimo de serpente, porém, cientificamente a palavra cobra refere-se às serpentes da família Colubridae (colubrídeos)." ${ }^{5}$ Obviamente que cobra e serpente não são a mesma coisa. Às vezes, é aceitável substituir um hiperônimo por um hipônimo, neste caso, substituir serpente com cobra, mas estes dois tipos de serpentes têm caraterísticas muito diferentes, vivendo em meios ecológicos diferentes. A víbora vive principalmente em zonas áridas e desérticas ou em zonas de montanha rochosa, enquanto que a cobra gosta de viver em zonas de bosque ou floresta.

As estratégias para traduzir os textos sobre os dois tipos de pássaros e répteis mencionados no livro são diferentes: no caso de 'estorninho', informações adicionais foram disponibilizadas aos leitores com vistas a melhor compreenderem os sentimentos descritos no poema; no caso de 'serpente', nenhuma informação adicional foi disponibilizada e a linha do poema não foi traduzida para manter uma imagem vívida de uma serpente a fazer uma siesta depois de uma suculenta refeição.

5 https://www.estudopratico.com.br/diferenca-em-cobra-serpente-e-vibora/. 


\subsection{O ambiente social}

Sendo um poeta experimental, talentoso e responsivo, Edwin Morgan pensou e discutiu diversos temas na sua poesia: da Terra ao espaço exterior, da história à cultura, da Escócia a países estrangeiros, entre outros. Neste trecho, dois aspectos são selecionados: o pano de fundo social e as figuras sociais.

\subsubsection{Pano de fundo social}

Edwin Morgan escreveu muitos poemas sobre a vida social da época, tais como os bairros pobres de Glasgow, a colocação em órbita lunar de uma nave soviética em 3 de novembro de 1957 com a famosa cadela Laika, a história da Escócia, etc. Avaliando o soneto intitulado 'Slate' ('Ardósia') que explora os contos sobre a história da Escócia, James McGonigal escreve:

Assim, o poeta [Edwin Morgan] está apontando não apenas para a vastidão desconhecida do espaço-tempo em que se encontra a Escócia, mas também talvez para a natureza paradoxal deste país, que é antigo em termos geológicos, e também bastante antigo em termos políticos (uma nação duramente conquistada nas Guerras da Independência há sete séculos), mas, por outro lado, relativamente recentemente, com o Ato de União de 1707, tendo-se tornado parte de um reino 'unido' maior.. ${ }^{6}$ (MCGONIGAL, 2013, p. 55)

Nesta frase longa existem dois elementos culturais relacionados com a história da Escócia, nomeadamente, 'the Wars of Independence' ('as Guerras da Independência') e 'the Act of Union of 1707' ('o Ato de União de 1707'). É necessário que os leitores chineses tenham os conhecimentos correspondentes a tais fatos históricos para entenderem a complexidade causal relativamente a tais eventos da história da Escócia. Por isso, a tradutora adicionou como nota de rodapé informação sobre as Guerras da Independência durante o fim do século XIII e o começo do século XIV entre a Inglaterra e a Escócia, resultando na in-

6 O texto de partida: So the poet [Edwin Morgan] is pointing out not only to the unknown vastness of space-time in which Scotland stands, but also perhaps to this country's paradoxical nature, which is ancient in geological terms, and also fairly old in political terms (a hard-won nationhood was attained in the Wars of Independence seven centuries ago), but which relatively recently, with the Act of Union of 1707, had become part of a larger 'united' kingdom. 
dependência da Escócia, e sobre o Ato de União de 1707 assinado entre as duas partes beligerantes para formarem um reino 'unido' maior.

\subsubsection{Figuras Diversas}

São incluídos nos poemas de Edwin Morgan diferentes tipos de personagens de vários países: escritores, pintores, músicos, historiadores, figuras da mitologia grega, figuras religiosas, políticos, entre outros. Principalmente, adicionei como nota de rodapé as informações relativas aos contextos em que as figuras são mencionadas, algumas das quais são curtas e simples, outras mais longas e complexas. Por exemplo, para duas pessoas mencionadas no livro, Pablo Neruda e Petrarch, as informações adicionadas como nota de rodapé são diferentes em termos de conteúdo e complexidade. No livro, Pablo Neruda é citado porque Edwin Morgan traduziu um poema de Pablo Neruda (MCGONIGAL, 2013, p. 62). Na tradução, as informações que adicionei sobre este autor incluíram o seu período de vida (1904-1973), nacionalidade (Chileno), identidade (diplomata e poeta) e atribuição do Prêmio Nobel de Literatura em 1971. Entretanto, o nome Petrarch é usado pela primeira vez no livro como uma referência de outro autor, Maurice Scève:

No seu isolamento [de Edwin Morgan] pós-guerra, foi particularmente atraído pela poesia de Maurice Scève (c. 1500-1564), um poeta renascentista que escreveu à maneira petrarquiana em que o objecto do amor do poeta é distante ou inatingível.7 (MCGONIGAL, 2013, p. 64)

Neste contexto, os leitores chineses têm de saber a história de vida relativa a Petrarch para entender o amor distante e inatingível relacionado com Petrarch. Por isso, além das informações sobre o seu período de vida (1304-1374), nacionalidade (Italiano), identidade (poeta), incluí também na tradução, como nota de rodapé, a história que mencionava Petrarch como tendo visto uma mulher chamada Laura em 1327 num espetáculo dela e desde então ficou apaixonado por ela e decidiu adorá-la à distância.

7 O texto de partida: In his [Edwin Morgan's] post-war isolation, he was particularly drawn to the poetry of Maurice Scève (c. 1500-1564), a Renaissance poet who wrote in the Petrarchan manner where the object of the poet's love is distant or unobtainable. 


\subsection{Imprecisão, falta de clareza, e inconsistência na estrutura de escrita}

Durante o processo de tradução, é possível que um tradutor, como um dos leitores mais cuidadosos do texto de partida, encontre alguma coisa que não está muito corretamente ou claramente escrita. Neste caso, o que pode um tradutor fazer? Manter-se fiel ao texto ou fiel à realidade? E como pode realizar-se a fidelidade?

É universalmente reconhecido que a tradução não é só uma transferência linguística. Segundo os teóricos da abordagem funcionalista, a tradução é um processo intercultural e comunicativo, envolvendo uma série de participantes como iniciador, comissário, produtor do texto original, produtor do texto alvo, utilizador do texto alvo e recipiente final do texto alvo (REISS, 2014; NORD, 2006). O escopo desta tradução do livro é para promover os conhecimentos dos chineses sobre a literatura e cultura da Escócia, bem como sobre o poeta Edwin Morgan. No livro existem elementos imprecisos e pouco claros que podem ter impactos negativos na compreensão para os leitores chineses. A seguir, alguns exemplos deste tipo são escolhidos para mostrar a mediação possível de um tradutor durante o processo de tradução.

No terceiro capítulo do livro, o autor James McGonigal citou um poema concreto de Edwin Morgan intitulado 'Message Clear' ('Mensagem Clara') para demonstrar o tema religioso-- 'I am the resurrection and the life' ('Sou a ressurreição e a vida'). Esta frase é da Bíblia, Evangelho de São João 11: $25^{8}$, mas no livro, foi escrito 5: 25 (MCGONIGAL, 2013, p. 15). Obviamente é um erro. Neste caso, a tradutora escolhou ser fiel ao fato ao invés de fiel ao autor, por isso, na tradução, corrigiu o erro, substituindo o número 5 pelo número 11.

Em comparação a erros relativamente óbvios, elementos em que falta clareza são mais desafiadores para um tradutor. No terceiro capítulo do livro, o autor falou sobre uma coleção de poemas chamada 'The Second Life' ('A Segunda Vida'), no qual inclui o poema 'Canedolia: An off-concrete Scotch Fantasia'. 'Caledonia' é o antigo nome para a Escócia. Isto é um poema experimental no qual Edwin Morgan usou os nomes de sítios na Escócia. No livro apenas alguns excertos do poema, às vezes, algumas palavras e linhas, são tiradas para explicar o significado deste poema, como na frase seguinte:

8 https://www.bibliaonline.com.br/acf/jo/11/21-26. 
Não é apenas um poema divertido em performance, mas também exprime de forma clara uma tensão peculiarmente escocesa entre os aspectos mais depressivos deste país e a cultura (“shiskine, scrabster, e snizort”), bem como a beleza frequentemente surpreendente da sua clara luz: 'blinkbonny! airgold! thundergay!’’ (MCGONIGAL 2013, p. 22)

Como leitora chinesa, não é difícil entender o poema em si por causa dos elementos culturais embutidos; como tradutora, a frase citada, com as ideias altamente condensadas, foi um grande desafio. Dois versos citados pelo autor, nomeadamente, 'shiskine, scrabster, and snizort' e 'blinkbonny! airgold! thundergay!', são parcialmente tiradas do poema mencionado. Entre estas linhas, seis palavras, nomeadamente, shiskine, scrabster, snizort, blinkbonny, airgold, e thundergay, por um lado, são nomes de seis lugares na Escócia; por outro lado, significam 'aspectos deprimentes deste país e desta cultura' e 'a beleza surpreendente da luz clara', como expressadas na frase. Mas em que sentido, por exemplo, estas três palavras como shiskine, scrabster e snizort podem mostrar os aspectos deprimentes da Escócia e a cultura dela? E porquê? Se as dúvidas não forem tiradas, a frase não fará sentido para os leitores chineses. A tradução não é um processo passivo de um tradutor, mas um processo de comunicação. Neste caso, a tradutora pediu ajuda a um amigo, que é também o iniciador do projeto de tradução, e ao mesmo tempo, um amigo do autor James McGonigal. O amigo explicou com mais pormenores os significados destas palavras, e depois reescreveu e expandiu a frase em três frases do seguinte modo:

Não é apenas um poema divertivo na performance, mas os nomes dos locais sugerem também uma tensão peculiarmente escocesa entre os aspectos mais depressivos do país e da cultura, como também a beleza muitas vezes surpreendente da sua clara luz. Nomes dos locais como 'shiskine, scrabster e snizort' evocam o som sibilante da chuva, e ecoam palavras negativas como 'crabs' (caranguejos), 'scab' (sarna), 'snort' (bufar) e 'drizzle' (chova fina). Alguns nomes são feitos de compostos como 'blinkbonny', 'airgold' e 'thundergay' que têm associações mais positivas, tais como 'bonny' (bonito), 'gold' (ouro) e 'gay' (feliz). ${ }^{10}$

9 O texto de partida: It is not only a funny poem in performance, but also neatly expresses a peculiarly Scottish tension between the most depressing aspects of this country and culture ('shiskine, scrabster, and snizort') and the often astonishing beauty of its clear light: 'blinkbonny! airgold! thundergay!.

$10 \mathrm{O}$ texto de partida: It is not only a funny poem in performance, but the place-names also suggest a peculiarly Scottish tension between the most depressing aspects of the country and culture, and the often astonishing beauty of its clear light. Place-names like 'shiskine, scrabster and snizort' evoke the hissing 
Segundo Andre Lefevere (2006/2016), a tradução é um processo de reescrita e manipulação. Com base na reescrita da frase no texto de partida feita pelo amigo, acrescentei as informações sobre o significado desagradável de chuva implicado nas palavras de shiskine, scrabster e snizort e as associações negativas relativas às palavras 'crabs' (caranguejos), 'scab' (sarna), 'snort'(bufar) e 'drizzle'(chuva fina), e as associações positivas relativas às palavras 'bonny' (pretty, bonito), 'gold' (ouro) e 'gay' (happy, feliz), e consegui fazer uma versão mais compreensível para os leitores chineses. E ao mesmo tempo, o processo de encontrar a solução para a dificuldade de tradução revela a comunicação entre a tradutora e o comissário, destacando a natureza da tradução como uma atividade de comunicação.

O livro foi escrito e orientado para os estudantes secundários e universitários na Escócia que já têm bastantes conhecimentos sobre a cultura e literatura escocesa. Muitos autores escoceses e internacionais são mencionados no livro, sem pormenores tais como os períodos de vida e os principais contributos literários destas pessoas. Mas o autor adicionou os detalhes para algumas figuras, por exemplo, os períodos de vida para Hugh MacDiarmid e Alexander Trocchi foram complementados nos parênteses no texto principal (MCGONIGAL, 2013, p. 20), entretanto, para os outros autores mencionados, tais como Allen Ginsberg e Jack Kerouac, nenhuma informação extra foi adicionada. Ignoro por que James McGonigal escolheu oferecer mais informações para alguns autores. Os leitores potenciais têm mais conhecimento sobre Allen Ginsberg e Jack Kerouac do que sobre Hugh MacDiarmid e Alexander Trocchi? Ou Hugh MacDiarmid e Alexander Trocchi são autores mais importantes na Escócia? Ou foi uma decisão puramente acidental? Seja qual for a razão, para os leitores chineses que não possuem conhecimento suficiente sobre esses autores, as passagens que os citam serão um grande desafio na leitura. Por isso, como tradutora, achei por bem facilitar o processo de leitura dos chineses através da oferta de mais informações. Nesse caso, os períodos de vida de todos os autores mencionados foram adicionados como notas de rodapé, e os períodos de vida de Hugh MacDiarmid e Alexander Trocchi, que estavam entre parênteses no corpo do texto, foram tirados e recolocados como notas de rodapé. Mesmo que seja uma pequena manipulação do texto original em termos de estrutura, para

sound of rain, and echo negative words like 'crabs', 'scab', 'snort' and 'drizzle'. Some place-names are made of compounds like 'blinkbonny', 'airgold' and 'thundergay' which have more positive associations, such as 'bonny' ('pretty'), 'gold' and 'gay'. 
os leitores chineses o livro ficará mais consistente na estrutura de escrita, em comparação com a situação anterior, em que as informações para alguns autores eram oferecidas, e para os outros, nada havia.

\subsection{Tradução do intraduzível, os elementos difíceis para traduzir}

Sendo um poeta extremamente experimental, Edwin Morgan escreveu muitos tipos de poesia abrangendo vários temas como amor, religião, exploração espacial e tecnologia e formas diversificadas, como poema concreto e poema sonoro.

O debate sobre a intraduzibilidade, em particular na área de poesia, tem uma história muito longa. O fato de muita poesia já ter sido traduzida, em certo sentido, evidencia a traduzibilidade da poesia, embora a tradução desse gênero não seja fácil. No trecho seguinte, são escolhidos alguns exemplos relativos aos títulos e versos de poemas para ilustrar as estratégias de tradução relacionadas com os elementos que parecem intraduzíveis.

\subsubsection{Preservação do texto fonte}

Alguns pesquisadores discutem a preservação do texto fonte como uma das estratégias tradutórias, por exemplo, Francis Henrik Aubert (1998, p. 106) definiu essa estratégia como 'empréstimo', o que 'é um segmento textual do Texto Fonte [texto de partida] reproduzido no Texto Meta [texto de chegada] com ou sem marcadores específicos de empréstimo (aspas, itálico, negrito, etc.)'. Em comparação com outras estratégias discutidas acima, parece que a preservação do texto fonte não é suficientemente discutida; contudo, esta prática de tradução tem sido adotada por tradutores para tratarem dos elementos difíceis ou intraduzíveis, em particular, em relação à poesia. Por exemplo, Virna Teixeira, tradutora brasileira manteve não traduzida a linha 's sz sz SZ sz SZ sz ZS zs ZS zs zs z' do poema chamado 'Siesta of a Hungarian Snake'. E isto não é único caso. Zhang Fenling (張芬齡), a mulher e tradutora de Chen Li (陳黎), um poeta de Taiwan, famoso pela escrita de poesia concreta, traduzia o poema 'A War Symphony' ('Uma Sinfonia de Guerra') de Chinês para Inglês de uma forma mesma, ou seja, o poema fica não traduzido, só o título foi traduzido (BRUNO, 2012, p. 258).

Baseada no uso frequente de duas consoantes 's' e ' $z$ ', o verso 's sz sz SZ sz SZ sz ZS zs ZS zs zs z’ de Edwin Morgan descreve de uma forma vívida o 
corpo de uma serpente após uma refeição reconfortante. Este poema pode ser classificado como uma poesia sonora e concreta. A poesia sonora, também chamada a poesia fonética ou poesia acústica, é um tipo de poesia oral. Remontando ao século IV na antiga China, a poesia concreta é um tipo de poesia vanguardista, experimental e principalmente visual, 'opera uma atualização radical dos recursos materiais (métrica, rima, aliteração, paronomásia, cortes e repetições de frase, neologismos, inversões sintáticas, plasticidade da letra impressa etc.) que se encontram dispersos e rarefeitos na poesia tradicional' (SIMON e DANTAS, 1982, p. 7). A poesia concreta apareceu na década de 1950 no Brasil e na Suíça, tendo sido primeiramente cunhada por Augusto de Campos na revista Noigandres de número 2, de 1955. Edwin Morgan se familiarizou com os poetas concretos brasileiros na década de 1960 e, durante essa década, tornou-se um dos principais tradutores para o inglês das obras dos irmãos Campos. (cf: CORBETT, 2015/2019; TEIXEIRA, 2019).

Mesmo que seja possível encontrar algumas palavras chinesas para transferir os sons das consoantes, o significado do título vai ficar perdido, por que a relação com a língua húngara estará cortada, o que no final faz o poema perder a coerência entre a língua húngara e a serpente. Portanto, decidi manter o verso não traduzido na tradução para o chinês.

Além de ser adotada para traduzir o conteúdo de um poema, esta estratégia pode ser também usada, completa ou parcialmente, na tradução de títulos de poemas. 'The New Divan' é um longo poema composto por cem estrofes. A palavra 'Divan' proveniente do árabe pode ser utilizada para significar três coisas diferentes: o conselho estadual, um sofá / uma cama, ou uma coleção de poemas. Todos significados são representados no poema. Neste caso, não é apropriado traduzir todos os três significados ou algum significado dos três no título do poema. No final, a tradutora decidiu manter a palavra 'Divan' não traduzida no título do poema. Por um lado, é um caminho sem saída; por outro lado, a manutenção da palavra faz lembrar a sua origem estrangeira, chamando atenção especial à sonoridade e significação da palavra.

\subsubsection{Tradução literal com nota de rodapé}

O poema intitulado 'Opening the Cage' também é de tradução muito difícil por causa de dois aspectos de significado envolvidos na palavra 'Cage'. 'Opening the Cage' é um poema composto por 14 variações baseadas numa frase de John Cage, músico e compositor americano - 'I have nothing to say and I am saying it 
and that is poetry', mostrando o espírito de otimismo nos anos sessenta do século passado. John Cage criou uma peça de música chamada 4'33" em que os músicos não tocaram nenhuma nota musical num período de 4 minutos e 33 segundos, apenas só silêncio, atraindo as pessoas a prestar atenção ao silêncio dos arredores. Por isso, 'Cage' no título deste poema está primeiramente relacionado com John Cage, mas ao mesmo tempo, 'cage' também significa 'cela', ou alguma coisa restritiva. Entretanto na tradução do título, não é possível incluir esses dois aspectos; então é preciso encontrar uma maneira melhor para expressar os dois significados sem tornar o título estranho e longo. No final, levando a colocação da palavra 'cage' com 'open' (abrir) em consideração, a tradutora decidiu manter o significado de 'cela' no título em si, mas adicionando, em nota de rodapé, a informação sobre o fundo relacionado com a criação da música 4'33”, uma experiência de que o próprio John Cage foi convidado a visitar e experimentar: a câmara anecoica de Harvard, em Cambridge, em 1951. No âmbito de silêncio completo, ele sentiu dois tipos de som, um sendo alto, e outro baixo. Para os leitores chineses, é interessante saber que John Cage foi inspirado pela filosofia ocidental, Budismo Chan/Zen chinês em particular, porque 'seu estudo do Budismo Zen mostrava-lhe a necessidade de ir além dos pares de opostos. ${ }^{11}$ Tais conhecimentos são necessários para atingir uma compreensão melhor do poema, e ao mesmo tempo podem reduzir a distância entre os leitores chineses, o músico e o poeta por causa da ligação com a filosofia chinesa relacionada.

\subsubsection{Explicação}

Além das estratégias mencionadas para lidar com os intraduzíveis, existe uma outra maneira: a explicação, ou seja, segundo Nord (2006), a transformação das informações implícitas do texto fonte numa informação textual explícita.

No poema intitulado 'A View of Things' ('Uma Visão das Coisas'), Edwin Morgan listou 22 tipos de amor e ódio para mostrar a variedade de vida e uma das 22 variedades é

what I love about newspapers is their etaoin shrdl... (MCGONIGAL, 2013, p. 32)

(o que amo sobre jornais é os seus etaoin shrdl...)

11 https://medium.com/zumbido/john-cage-e-o-sil\%C3\%AAncio-a6a4078c789c. 
As últimas palavras 'etaoin' e 'shrdl' foram criadas por Edwin Morgan, significando os erros tipográficos nos jornais na época de composição tipográfica em metal quente. É óbvio que não existem equivalentes dessas 'palavras' em chinês. Se essas 'palavras' de combinação aleatória de letras se mantiverem na tradução chinesa, os leitores chineses não conseguirão entender o significado. Nesse caso, é necessário encontrar outra maneira para ajudar os leitores chineses a perceber o que o poeta quer dizer. Com esta ideia em mente, a tradutora preferiu fazer uma explicação na tradução, nomeadamente, essas duas palavras são traduzidas por uma locução que tem o significado de 'erros tipográficos'.

\section{Conclusão}

Os elementos culturais são grandes desafios para os tradutores. As estratégias discutidas acima nos vários aspectos dos elementos culturais, seja uma adição no corpo do texto ou uma amplificação como nota de rodapé, seja a preservação de texto fonte ou uma explicação mais circunstanciada e clara, demonstram a natureza da tradução como um processo de reescrita, manipulação, mediação e comunicação, no qual o tradutor ou tradutora tem de pensar e adotar várias estratégias, e funcionar como mediador entre autor e os leitores-alvo para concretizar o escopo de uma comunicação intercultural. Neste estudo de caso, a autora demonstrou como essas preocupações se aplicam à tradução de um guia de estudo introdutório sobre alguém que é uma figura nacional num país, mas relativamente desconhecido noutro. Edwin Morgan, tradutor de muitos poetas importantes de vários países, incluindo os irmãos Campos, é um poeta considerável por mérito próprio e merece ser mais conhecido na China. À primeira vista, esse objetivo parece ser atendido pela tradução de um guia de estudo destinado a alunos mais velhos da escola escocesa, seus professores e meio acadêmico universitário. No entanto, como vimos, mesmo uma tradução tão simples como a explanada antes exige apurados processos de manipulação e transformação antes de ser considerada apropriada à compreensão dos leitores chineses. 


\section{Referências bibliográficas}

BAKER, Mona. In other words: A course book on translation. London: Routledge, 1992.

BRUNO, Cosima. Words by the Look: Issues in Translating Chinese Visual Poetry. Leiden: Brill, 2012.

CHESTERMAN, Andrew. Memes of translation: the spread of ideas in translation theory. Amsterdam and Philadelphia: John Benjamins, 1997/2016.

CORBETT, John. “Concrete Realities.” In: RIACH, Alan (Org.). The International Companion to Edwin Morgan. Glasgow: Association for Scottish Literary Studies, 2015, p. 130-144.

CORBETT, John. "Mapping the International Concrete Poetry Network.” In: CORBET'T, John and HUANG, Ting (Orgs.). The Translation and Transmission of Concrete Poetry. London: Routledge, 2019.

AUBERT, Francis Henrik. "Modalidades de tradução: teoria e resultados." Tradterm, v. 5, n. 1, 1998, p. 99-128/129.

HERMANS, Theo. The Manipulation of Literature: Studies in Literary Translation. London: Croom Helm, 1985.

KATAN, David. Translating Cultures. An Introduction for Translators, Interpreters and Mediators. Manchester: St. Jerome, 1999.

KELLY, Dorothy. A bandbook for translator trainers: A guide to reflective practice. Manchester: St. Jerome, 2005.

LEFEVERE, Andre. Translation, rewriting, and the manipulation of literary fame. London: Routledge, 2006/2016.

MCGONIGAL, James. The Poetry of Edwin Morgan. Glasgow: Association for Scottish Literary Studies, 2013.

MORGAN, Edwin. Collected Poems. Manchester: Carcanet, 1990.

MUNDAY, Jeremy. Introducing Translation Studies: theories and applications. London/ New York: Routledge, 2016.

NEWMARK, Peter. A Textbook of Translation. London/New York: Prentice Hall, 1988.

NORD, Christiane. Translating as a purposeful activity: Functionalist approaches explained. Shanghai: Shanghai Foreign Language Education Press, 2001.

NORD, Christiane. "Translating for Communicative Purposes across Culture Boundaries". Journal of Translation Studies, v. 9 , n. 1, 2006, p. 43-60.

REISS, Katharina. \& VERMEER, Hans. Towards a General Theory of Translational Action: Skopos Theory Explained. London: Taylor \& Francis, 2014.

SIMON, Iumna Maria e DANTAS, Vinicius. Poesia Concreta. São Paulo: Literatura Comentada, 1982.

TEIXEIRA, Virna. (trans.). Edwin Morgan: Na Estação Central. Brasília: Universidade de Brasília, 2006. 
TEIXEIRA, Virna. "Haroldo de Campos na Escócia nos Anos 1960 e o McConcretismo". GUERINI, Andréia, COSTA, Walter. Carlos, e HOMEM de Mello, Simon. Haroldo de Campos, Tradutor e Traduzido. São Paulo: Editora Perspectiva, 2019, p. 285-292. 